S.O.Schoenberg ${ }^{1}$ M.V.Knopp ${ }^{1} \cdot$ M. Bock $\cdot$ F. Floemer ${ }^{1} \cdot$ F. Kallinowski ${ }^{2} \cdot$ M. Essig $^{1}$

H. Hawighorst ${ }^{1}$ - A. Just ${ }^{3} \cdot$ G. Laub ${ }^{4} \cdot$ M.R. Prince ${ }^{5} \cdot$ G. van Kaick ${ }^{1}$

${ }^{1}$ Deutsches Krebsforschungszentrum, Forschungsschwerpunkt radiologische Diagnostik

und Therapie, Heidelberg. ${ }^{2}$ Chirurgische Universitätsklinik, Heidelberg $\cdot{ }^{3}$ I. Physiologisches Institut, Heidelberg $\cdot{ }^{4}$ Siemens Medizintechnik, Erlangen $\cdot{ }^{5}$ Department of Radiology, University of Michigan, Ann Arbor, Michigan, USA

\section{MR-Bildgebung der Nieren} Neue Ansätze in der Diagnostik*

\section{Zusammenfassung}

Fragestellung: Darstellung neuer diagnostischer Möglichkeiten im Bereich der Niere mittels schneller Magnetresonanz (MR)Bildgebung.

Methodik: Vorgestellt wird ein kombiniertes morphologisches und funktionelles Untersuchungskonzept bestehend aus schneller morphologischer Bildgebung, multiphasischer 3D-Gadolinium-MR-Angiographie, MR-Urographie und MR-Flußmessung.In einer einzigen MR-Untersuchung sollen vaskuläre Erkrankungen erfaßt, eingestuft und auf ihre hämodynamische und funktionelle Signifikanz überprüft werden, renale Läsionen detektiert und differenziert sowie die Harnabflußwege beurteilt werden.

Ergebnisse: Durch Integration der gewonnenen morphologischen und funktionellen Daten lassen sich Nierenarterienstenosen, benigne und maligne renale Tumoren, Harnabflußstörungen und kongenitale Fehlbildungen mit ähnlicher Genauigkeit wie in den konventionellen radiologischen Verfahren erfassen. Der Nachteil der geringeren räumlichen Auflösung kann dabei weitgehend durch den besseren Gewebekontrast und die zusätzliche, funktionelle Information kompensiert werden.

Schlußfolgerung: Ein kombiniertes

MR-Untersuchungskonzept stellt ein zuverlässiges, nicht-invasives und kosteneffektives Verfahren zur umfassenden diagnostischen Abklärung der Niere dar.

\section{Schlüsselwörter}

Magnetresonanzangiographie ·

Magnetresonanzurographie · Niere ·

Phasenkontrast · Schnelle Bildgebung ie Magnetresonanztomographie war in ihrer Anwendung im Abdomen ursprünglich durch lange Meßzeiten, geringe Ortsauflösung und vielfältige Artefakte begrenzt. Frühzeitig wurde jedoch die Attraktivität dieser Methode erkannt, da neben der reinen morphologischen Beurteilung auch eine Vielzahl unterschiedlichster funktioneller Information gewonnen werden kann. Weitere Vorteile der Magnetresonanztomographie liegen in der fehlenden Strahlenexposition sowie in der Verwendung nicht nephrotoxischer Kontrastmittel. Bis vor kurzem ließen sich diese verschiedenen diagnostischen Teilaspekte aufgrund der langen Untersuchungszeiten nicht in ein umfassendes Untersuchungskonzept integrieren. Erst durch eine Reihe neuer Entwicklungen konnte diese realisiert werden. Hierzu gehören vor allem die Entwicklung sehr leistungsfähiger Gradientensysteme, die sehr kurze Meßzeiten ermöglichen [ 1 , 2] und die Einführung neuartiger Kontrastmittel, die die Bildqualität auch bei sehr kurzen Meßzeiten verbessern und neues Potential für funktionelle Untersuchungen eröffnen [3]. Durch eine kombinierte morphologische und funktionelle Beurteilung der Niere in einer einzigen MR-Untersuchung läßt sich nach Integration aller gewonnenen Teilinformationen eine hohe diagnostische Aussagekraft im ganzen Spektrum renaler Erkrankungen erzielen. Von großer Bedeutung ist hierbei die Standardisierung der Untersuchung, der Datenauswertung und der Befundung [4]. Im folgenden soll ein klinisch validiertes Untersuchungskonzept vorge- stellt und anhand von unterschiedlichen renalen Pathologien beleuchtet werden. Hierzu zählen insbesondere die Erkennung renovaskulärer Erkrankungen, vor allem der Nierenarterienstenose mit der präoperativen Einstufung der hämodynamischen und funktionellen Signifikanz sowie der Beurteilung des postoperativen Revaskularisationserfolges. Daneben hat die Magnetresonanztomographie einen zunehmenden Stellenwert bei der Darstellung urodynamisch wirksamer Pathologien des Harnabflußtraktes. Schließlich kommt der Technik aufgrund des überlegenen Weichteilkontrastes auch eine wichtige Rolle bei der Differenzierung und Staging von Nierentumoren zu.

\section{Material und Methoden}

Das von uns verwendete Untersuchungskonzept soll in einer MR-Untersuchung unterschiedliche Fragestellungen in den verschiedenen Teilregionen des renalen Systems abklären. Hierzu gehören die:

- Erfassung vaskulärer Erkrankungen, insbesondere Nierenarterienstenosen,

\footnotetext{
* Diese Arbeit wurde z.T. durch Förderungen der "Deutschen Forschungsgemeinschaft", dem „Verein zur Förderung der Krebserkennung und Krebsvorsorge, e.V." und dem „Tumorzentrum Heidelberg/Mannheim" ermöglicht
}

Dr.S.O.Schoenberg Deutsches Krebsforschungszentrum (DKFZ), Abteilung Radiologische Diagnostik und Therapie, Im Neuenheimer Feld 280, D-69120 Heidelberg 
Radiologe

1999 · 39:373-385 @ Springer-Verlag 1999

S.O.Schoenberg · M.V. Knopp $\cdot$ M. Bock

F. Floemer $\cdot$ F. Kallinowski $\cdot$ M. Essig

H. Hawighorst · A. Just · G. Laub · M.R. Prince

G.van Kaick

\section{MR imaging of the kidneys: new diagnostic strategies}

\section{Summary}

Aim: New diagnostic strategies for evaluation of the kidney by fast MR imaging techniques.

Material and methods: A comprehensive morphologic and functional evaluation of the kidney is proposed using fast MR imaging of renal morphology, multiphase 3D gadolinium MR angiography, MR urography and MR flow measurements. A single MR examination is designed to grade renovascular disease and assess the hemodynamic and functional significance, detect and characterize renal lesions and evaluate the urinary tract.

Results: The combined analysis of morphologic and functional data allows reliable assessment of renal artery stenosis, benign and malignant renal masses and diseases of the renal collecting system and ureters, as well as congenital abnormalities in good agreement to the results of conventional imaging modalities. The improved tissue contrast and additional functional information compensates for the disadvantage of a lower spatial resolution.

Conclusion: Combined morphologic and functional MR examination represents a reliable, non-invasive and cost-effective alternative imaging modality for comprehensive diagnostic evaluation of renal disease.

\section{Key words}

Magnetic resonance angiography · Magnetic resonance urography · Kidney ·

Phase-contrast · Fast imagin

\section{Nierendiagnostik}

und deren hämodynamische und funktionelle Signifikanz,

- Detektion einer renalen Läsion, Charakterisierung ihrer Dignität und möglichst genauer Beschreibung ihrer Ausdehnung,

- Erfassung der lokoregionären Tumorausbreitung,

- Beurteilung der Harnabflußwege.

Alle Untersuchungen wurden auf einem 1,5T Magnetom Vision Tomographen (Siemens, Erlangen) durchgeführt, der mit einem Echo-Planar-Imagingfähigen Gradientensystem ausgestattet ist (maximale Gradientenstärke: $25 \mathrm{mT} / \mathrm{m}$, kürzeste Anstiegszeit zum Maximum: $300 \mu \mathrm{s}$ ). Die Patienten wurden in einer Empfangsspule mit $4 \mathrm{Ar}$ ray-Elementen untersucht, die in Höhe der Nieren positioniert wurde. Folgende Techniken kommen dabei zur Anwendung:

\section{Multiphasische kontrastmittel- verstärkte dreidimensionale MR-Angiographie (3D-Gd-MRA)}

Mit der Einführung der kontrastmittelverstärkten $3 \mathrm{D}$-Gadolinium-MR-Angiographie (3D-Gd-MRA) [5] wurden die Nachteile der nativen MRA-Techniken, wie der Time-of-flight- und Phasenkontrastangiographie, überwunden. Insbesondere Artefakte wie Saturation in Schichtebene, Signaldephasierung und Atemartefakte treten bei der $3 \mathrm{D}-\mathrm{Gd}$ MRA fast nicht mehr auf. Erforderlich ist hierbei die Applikation von Gadolinium-DTPA in Bolusform, wobei die Ankunft des Kontrastmittels mit der Akquisition der zentralen Anteile des $\mathrm{k}$-Raums genau synchronisiert werden muß. Dafür stehen in Abhängigkeit vom MR-System unterschiedliche Verfahren zur Verfügung, wie die Testbolusmessung, automatisierte (MRSmartprep $\left.{ }^{\circledR}\right)$ sowie optisch kontrollierte (MR-Fluoroscopy ${ }^{\circledR}$ ) Detektion der Kontrastmittelanflutung. Eine methodische Weiterentwicklung stellt die multiphasische 3D-Gd-MRA dar [6]. Diese Technik erlaubt in einer einzigen Atemanhaltephase während der Passage eines Gadolinium-Kontrastmittelbolus die Akquisition von mehreren dreidimensionalen Datensätzen in der arteriellen, parenchymatösen und venösen Phase des renovaskulären Systems (Abb. 1a, 2). Eine zeitaufwendige Be- stimmung der Kontrastmitteltransitzeit zur Synchronisation der Kontrastmittelinfusion und Datenakquisition entfällt. Hiermit gelingt zum einen die zeitlich getrennte Darstellung von Angiogrammen der Nierenarterien, Nierenvenen sowie der Vena cava inferior in räumlich hoher Auflösung unter 2 $\mathrm{mm}$ in allen Raumrichtungen. Zusätzlich läßt sich hiermit in den Einzelschichten der dreidimensionalen Datensätze die Kontrastmittelanreicherungskinetik des Nierenparenchyms zeitaufgelöst erfassen. Während der renalen Exkretionsphase aufgenommene Datensätze mit Fettsuppression lassen sich sowohl zur morphologischen Beurteilung des Kelchsystems und der ableitenden Harnwege als auch zur funktionellen Analyse der Ausscheidung analog zur Röntgenurographie heranziehen [7] (Abb. 8).

Alternativ kommt hier auch die Saturations-Inversions-Projektions-(SIP)Technik zur Anwendung, die ebenfalls ein Urogram in einer einzigen Atemanhaltephase erstellt [8] (Abb. 7). Hierbei wird eine mehrere Zentimeter dicke zweidimensionale Schicht im Bereich des Harntraktes aufgenommen, in der durch geeignete Abfolge eines $90^{\circ}$ und $180^{\circ}$ Pulses sämtliches Gewebesignal unterdrückt wird und nur die mit Gadolinium kontrastierten Strukturen als Projektionsbild zur Darstellung kommen.

Zur Abklärung bestimmter angiographischer Fragestellungen ist es erforderlich, anstatt einer zeitlich aufgelösten Betrachtung die räumliche Auflösung zu maximieren (Abb. 4-6). Hierzu gehören die Abklärung einer fibromuskulären Dysplasie, vaskulärer $\mathrm{Pa}$ thologien im Kindesalter sowie von Transplantatnierenarterien. Mit leistungsfähigen Gradientensystemen kann hierbei unter Verwendung von Sequenzen mit 512er Matrix nach Interpolation der Daten eine Auflösung von unter einem Millimeter in allen Raumrichtungen erzielt werden, wobei Meßzeiten von etwa 25 bis 30 Sekunden zur Aufnahme eines einzigen 3D Datensatz erforderlich sind.

\section{Segmentierte echoplanare Cine-Phasenkontrastflußmessung}

Mit Hilfe der EKG-getriggerten CinePhasenkontrasttechnik ist es möglich, 

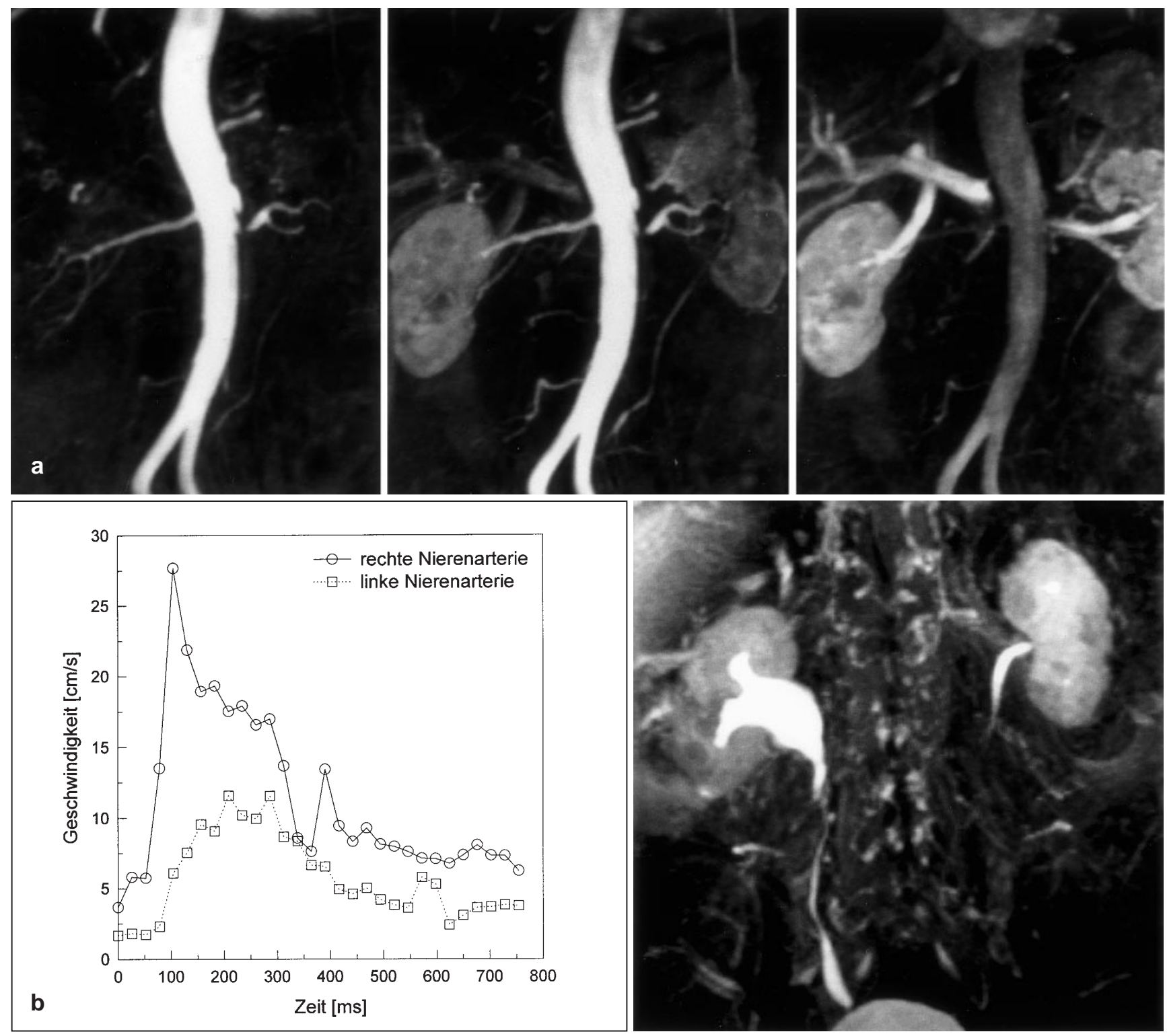

Abb. 1a-c $\Delta$ 63jähriger Patient mit Hypertonie und progressiver Niereninsuffizienz. a In der früharteriellen Phase der Multiphasenangiographie ( $T R=3,2 \mathrm{~ms}, T E=1,1 \mathrm{~ms}$, Aufnahmezeit für einen 3D Datensatz $=6,4 \mathrm{~s}$ ) Nachweis einer hochgradigen proximalen Nierenarterienstenose links. Im Vergleich zur Gegenseite verzögerte parenchymale Kontrastierung der geschrumpften linken Niere in der spätarteriellen Phase (Mitte) bei weitgehend gleicher Kontrastierung in der frühvenösen Phase (rechts). $b$ EKG-getriggerte Cine-Phasenkontrastflußmessung (FLASH 2D, TR=26 ms, TE=5 ms). Darstellung eines physiologischen Blutflußprofils in der rechten Nierenarterie mit erhaltenem frühsystolischen Peak bei einem arteriellen Mittelfluß von $310 \mathrm{ml} / \mathrm{min}$. Links stark abgeflachtes Flußprofil mit deutlich reduziertem arteriellen Mittelfluß von $93 \mathrm{ml} / \mathrm{min}$. C MR-Urographie (3D FLASH mit Fettunterdrückung, TR=5 ms, TE=2 ms, Voxelgröße $=1,4 \times 2 \times 2 \mathrm{~mm}$, Meßzeit= $27 \mathrm{~s}$ in Atemanhaltetechnik). Verzögerte Ausscheidung links mit Kontrastmittelretention der Niere und inkompletter Darstellung des Ureters. Diagnose: hämodynamisch und funktionell relevante Nierenarterienstenose links

in hoher zeitlicher und räumlicher Auflösung die Blutflußgeschwindigkeit zu bestimmen. Damit gelingt sowohl eine nicht-invasive Bestimmung der mittleren renalen Durchblutung als auch die Erfassung hämodynamischer Veränderungen. Die Meßgenauigkeit dieser
Technik wurde bereits in mehreren Studien am Phantom, Versuchstier und Patient validiert $[10,11]$. Um die hohe zeitliche Auflösung der Messung von weniger als $30 \mathrm{~ms}$ zu ermöglichen, erforderten die bisher zur Anwendung gekommenen FLASH-Techniken Untersu- chungszeiten von mehreren Minuten, was diese Methode zeitaufwendig und anfällig gegen Atemartefakte im Abdomen macht. Mit der Entwicklung einer segmentierten echoplanaren (EPI) Bildgebungstechnik kann die gleiche Messung gegenwärtig in einer einzigen Atemanhaltephase durchgeführt werden [12] (Abb. 3b). Dabei wird pro HFAnregung mehr als eine k-Raumzeile ausgelesen, wodurch sich die Meßzeit entsprechend verkürzen läßt. Entscheidend für die Anwendung solcher EKGgetriggerter Meßtechniken ist die Verwendung eines optisch entkoppelten EKG-Systems, das eine zuverlässige Triggerung auch unter Einfluß starker Gradienten erlaubt.

Mit diesen ultraschnellen Untersuchungstechniken gelingt in 4 Ateman- 


\section{Nierendiagnostik}
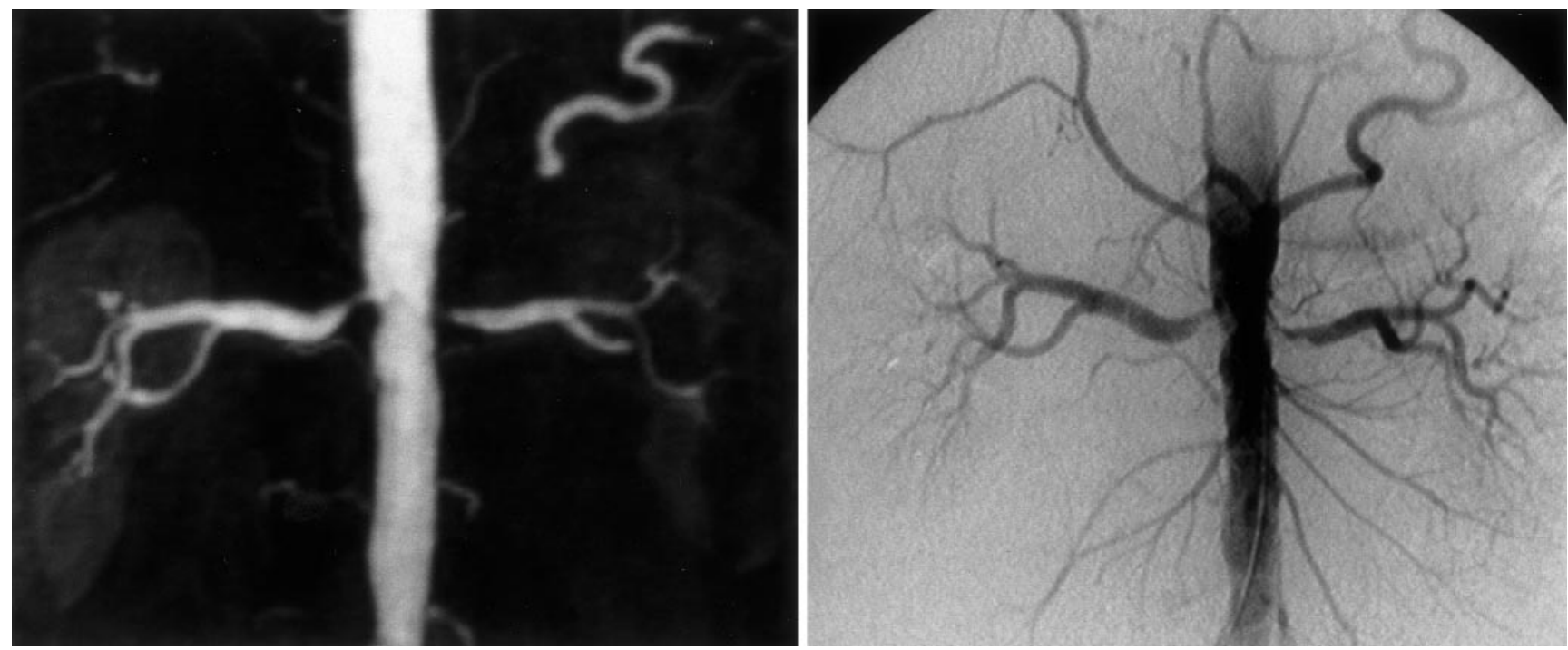

Abb. $2 \Delta$ Links: früharterielle Phase einer abdominellen Multiphasenangiographie (TR 3,2 ms, $\mathrm{TE}=1,1 \mathrm{~ms}$,Meßzeit 6,4 s, Voxelgröße 1,5 $\times 1,8 \times 1,8 \mathrm{~mm}$ ) bei einem 57jährigen Patienten mit bilateraler Nierenarterienstenose. Bei minimaler Parenchymkontrastierung lassen sich die Nierenarterien distal bis zur mittleren Segmentarterie in guter Übereinstimmung zur DSA (rechts) verfolgen. Diagnose: ostiale Nierenarterienstenose beidseits, rechts mittelgradig (70\%), links hochgradig (90\%, reproduziert mit freundlicher Genehmigung von Radiology)

haltephasen eine Darstellung der renalen Gefäßanatomie und der Harnabflußwege sowie eine Abschätzung der normalen Gewebeperfusion [13].

\section{Morphologische Bildgebung}

Aufgrund der kurzen Meßzeiten lassen sich die oben beschriebenen Methoden innerhalb einer Untersuchung mit MRStandardsequenzen zur morphologischen Beurteilung der Niere kombinieren. Koronar und sagittal in Atemanhaltetechnik durchgeführte T1-gewichtete fettunterdrückte Gradientenechosequenzen erlauben eine Bestimmung der Organgröße im Seitenvergleich. Renale Läsionen können bis zu einer minimalen Größe von etwa $1 \mathrm{~cm}$ zuverlässig erkannt werden, wobei die Schichtorientierung insbesondere die Beurteilung der krankiokaudalen Ausdehnung erleichtert.

Fettunterdrückte schnelle T2-gewichtete Bildgebungssequenzen erlauben in Meßzeiten von weniger als $30 \mathrm{~s}$ eine weiterführende Charakterisierung renaler Läsionen, die Beurteilung des peri- und pararenalen Raumes sowie der lokoregionären Lymphknotenstationen. Hierbei kommt der Verwendung der Fettunterdrückung eine besondere Rolle zu. Die frequenzselektive Absättigung der Fettprotonen ist im
Abdomen jedoch nicht immer einfach, da es hier auf Grund der verschiedenen Gewebeübergänge zu massiven Verzerrungen des statischen Magnetfeldes und damit zu stark unterschiedlichen Resonanzfrequenzen kommt. Durch Homogenisierung (Shim) können diese Verzerrungen nur teilweise behoben werden - daher ist besondere Sorgfalt bei der Applikation von Fettunterdrückungssequenzen und Interpretation fettunterdrückter Bilder geboten. Zur Anwendung kommen hier singleshot Turbospinecho-Sequenzen mit Half-Fourier-Rekonstruktion (HASTE, "half fourier single shot turbo spin
N.a., nicht angegeben echo“). Die räumliche Auflösung dieser Sequenzen ist allerdings auf 1 bis 2 $\mathrm{mm}$ begrenzt. Deutliche höhere Auflösungen bis 0,4 bis $0,5 \mathrm{~mm}^{2}$ in Schichtebene lassen sich mit Standard Multishot-Turbospinechosequenzen erzielen. Bei einer Schichtdicke von $5 \mathrm{~mm}$ ist die resultierende Voxelgröße bei besserem Gewebekontrast mit der Computertomographie vergleichbar. Nachteilig sind jedoch die langen Meßzeiten von mehreren Minuten und damit der deutlich größere Einfluß von Atemartefakten, die sich jedoch durch Verwendung mehrerer Akquisitionen sowie Atemtriggerung reduzieren lassen.

Koronar durchgeführte T2-gewichtete Messungen in Atemanhaltetechnik mit der HASTE-Sequenz ermöglichen außerdem bei extrem langen Echozeiten eine native Darstellung der flüssigkeitsgefüllten Anteile der Harnabflußwege.

\begin{tabular}{|c|c|c|c|c|c|}
\hline Autor & Jahr & Patienten (n) & Sensitivität & Spezifität & Stenosegrad \\
\hline Prince & 1995 & 19 & $100 \%$ & $93 \%$ & N.a. \\
\hline Grist & 1996 & 35 & $88 \%$ & $88 \%$ & $>50 \%$ \\
\hline Snidow & 1996 & 47 & $100 \%$ & $89 \%$ & N.a. \\
\hline Steffens & 1997 & 50 & $96 \%$ & $95 \%$ & N.a. \\
\hline Hany & 1997 & 39 & $93 \%$ & $98 \%$ & $>50 \%$ \\
\hline De Cobelli & 1997 & 55 & $100 \%$ & $97 \%$ & $>50 \%$ \\
\hline Bakker & 1998 & 54 & $97 \%$ & $92 \%$ & $\geq 50 \%$ \\
\hline Schoenberg & 1999 & 21 & $100 \%$ & $91 \%$ & $>50 \%$ \\
\hline
\end{tabular}



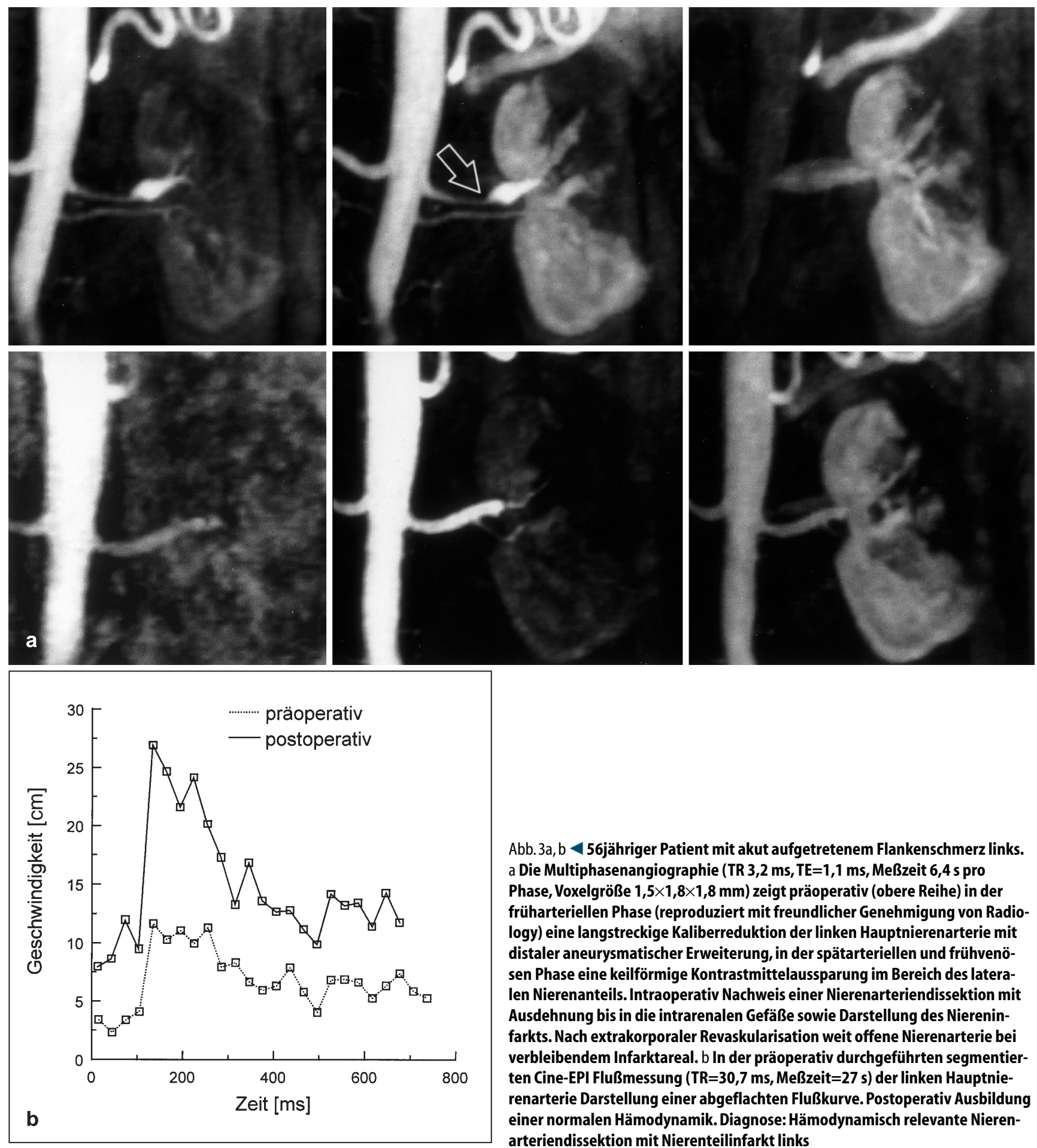

Abb. 3a, $b<$ 56jähriger Patient mit akut aufgetretenem Flankenschmerz links. a Die Multiphasenangiographie (TR 3,2 ms, TE=1,1 ms, Meßzeit 6,4 s pro Phase, Voxelgröße 1,5×1,8×1,8 mm) zeigt präoperativ (obere Reihe) in der früharteriellen Phase (reproduziert mit freundlicher Genehmigung von Radiology) eine langstreckige Kaliberreduktion der linken Hauptnierenarterie mit distaler aneurysmatischer Erweiterung, in der spätarteriellen und frühvenösen Phase eine keilförmige Kontrastmittelaussparung im Bereich des lateralen Nierenanteils. Intraoperativ Nachweis einer Nierenarteriendissektion mit Ausdehnung bis in die intrarenalen Gefäße sowie Darstellung des Niereninfarkts. Nach extrakorporaler Revaskularisation weit offene Nierenarterie bei verbleibendem Infarktareal. $b$ In der präoperativ durchgeführten segmentierten Cine-EPI Flußmessung ( $T R=30,7$ ms, Meßzeit=27 s) der linken Hauptnierenarterie Darstellung einer abgeflachten Flußkurve. Postoperativ Ausbildung einer normalen Hämodynamik. Diagnose: Hämodynamisch relevante Nierenarteriendissektion mit Nierenteilinfarkt links

\section{Ergebnisse}

\section{Nierenarterien}

Erfahrungen mit der $3 \mathrm{D}$-Gd-MRA liegen bereits aus zahlreichen Studien der letzten vier Jahre vor. Diese Studien kommen einheitlich zu hohen Sensitivitäten und Spezifitäten bei der Detektion von proximalen Nierenarterienste- nosen im Vergleich zur konventionellen digitalen Subtraktionsangiographie (Tabelle 1). Als Kriterium diente in diesen Studien ein vereinfachtes drei- bis vierstufiges Schema zur Stenosegradeinstufung $(0 \%,<50 \%, \geq 50 \%,>80 \%)$. Fehleinstufungen treten insbesondere bei höhergradigen Stenosen auf, da das verbleibende stenosierte Lumen häufig unterhalb der Auflösungsgrenze der
Technik liegt. Eine hundertprozentige Übereinstimmung zwischen $3 \mathrm{D}-\mathrm{Gd}-$ MRA und DSA ist nicht zu erwarten, da beide Techniken grundsätzlich methodische Unterschiede aufweisen: die 3DGd-MRA stellt eine echte dreidimensionale Technik mit geringer Auflösung dar, während die DSA als zweidimensionale Technik bei hoher Auflösung auf wenige Projektionen limitiert 

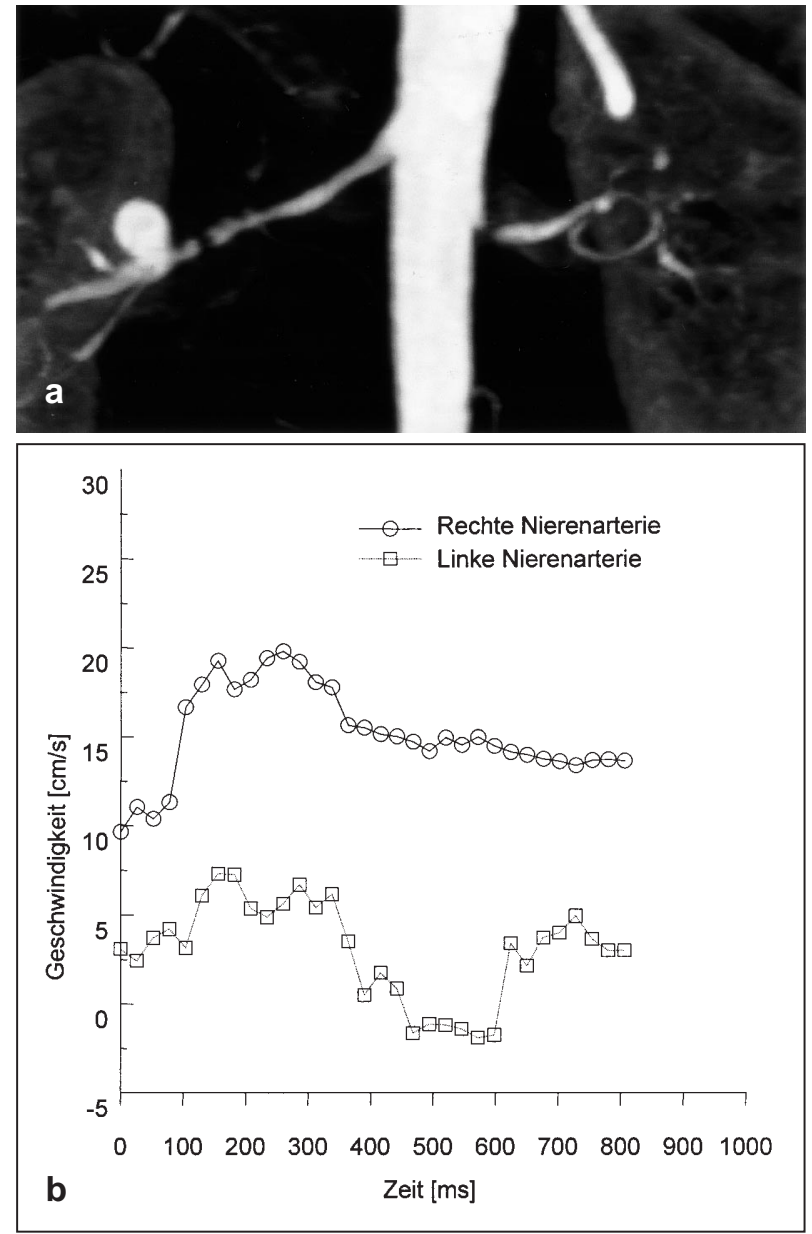

Abb. 4a, b $\Delta$ 52jährige Patientin mit fibromuskulärer Dysplasie, medialer Typ. a Die hochauflösende 3D-Gadolinium-MRA ( $T R=4,6 \mathrm{~ms}$, TE=1,8 ms, Voxelgröße 0,7 $\times 1,2 \times 1,2 \mathrm{~mm}$ ) zeigt multiple Kaliberschwankungen der rechten Nierenarterie, die charakteristischen perlschnurartigen Veränderungen sowie ein bifurkationsnahes Aneurysma. Links Nachweis einer proximalen hochgradigen Nierenarterienstenose. $b$ In der MR-Flußmessung (FLASH 2D, TR=26 ms, TE=5 ms) Teilverlust des frühsystolischen Peaks in der rechten Nierenarterie als Zeichen einer nur gering hämodynamisch wirksamen Stenosierung (Mittelfluß: $442 \mathrm{ml} / \mathrm{min}$ ). Links weitgehender Verlust der systolischen Geschwindigkeitsanteile mit retrogradem Fluß in der Endsystole (Mittelfluß: $63 \mathrm{ml} / \mathrm{min}$ ). Diagnose: hämodynamisch signifikante Nierenarterienstenose links

bleibt. Bis jetzt liegen keine Daten zur Genauigkeit der 3D-Gd-MRA bei der Detektion distaler und intrarenaler Stenosen vor, da hier die Gefäßvisualisierung aufgrund von Parenchymkontrastierung sowie Überlagerungen durch kontrastierte venöse Strukturen und des Kelchsystems deutlich geringer ist. Mit der multiphasischen 3D-Gd-MRA gelingt neben der zuverlässigen Einstufung proximaler Nierenarterienstenosen eine deutlich bessere Visualisierung der distalen Anteile der Nierenarterie bis zur zweiten Aufteilung (Abb. 2). Der Einfluß von Artefakten bei dieser Technik erwies sich als signifikant geringer im Vergleich $\mathrm{zu}$ herkömmlichen 3DGd-MRA-Techniken [14].
Die 3D-Gd-MRA eignet sich auch zur Diagnose der fibromuskulären Dysplasie (FMD). Unter Verwendung hochaufgelöster MR-Angiogramme werden auch die typischen perlschnurartigen Veränderungen bei der medialen FMD sichtbar in guter Übereinstimmung zur konventionellen DSA. Allerdings fehlen hier noch gesicherte Daten aus prospektiven Vergleichsstudien. Als besonders vorteilhaft erweist sich der dreidimensionale Charakter der 3D-Gd-MRA bei der Darstellung räumlich komplexer Strukturen wie dem Nierenarterienaneurysma (Abb. 4). Durch multiplanare Reformatierung des $3 \mathrm{D}$ Datensatzes läßt sich hierbei der Bezug zu den einzelnen Gefäßstrukturen optimal herausarbeiten. Dies ist vor allem diagnostisch bedeutsam, wenn Pathologien der abdominellen Aorta die Nierenarterie mit einbeziehen wie u.U. bei einer Aortendissektion [15].

Die Zuverlässigkeit der verschiedenen MR-Techniken hinsichtlich der Einstufung der hämodynamischen und funktionellen Signifikanz einer Stenose konnte in mehreren Studien klinisch validiert werden $[16,17]$. Hierbei wurden die präoperativ gefundenen hämodynamischen und funktionellen Einschränkungen mit dem postoperativen Veränderungen nach erfolgreicher Revaskularisation korreliert. Signifikante Nierenarterienstenosen können anhand einer charakteristischen Abflachung des zeitaufgelösten Blutflußprofils in der Cine-Phasenkontrastflußmessung sowie einer verzögerten Parenchymkontrastierung in der $3 \mathrm{D}-\mathrm{Gd}-$ MRA zuverlässig erkannt werden (Abb. $1,3 \mathrm{~b}, 4 \mathrm{~b}$ ). Insbesondere der Verlust des auch aus der Sonographie bekannten frühsystolischen Anstiegs der Blutflußgeschwindigkeit (frühsystolischer Peak) in der zeitaufgelösten Flußmessung dient als zuverlässiges Kriterium für eine beginnende hämodynamisch signifikante Störung. Die Analyse der mehrphasisch aufgenommenen 3D Datensätze läßt außerdem geringste Unterschiede in der Stärke der Parenchymkontrastierung im Seitenvergleich erkennen. Eine Sicherung des prädiktiven Wertes dieser Methoden in Bezug auf den Operationserfolg steht noch aus.

Die Erfassung morphologischer und funktioneller Information sowie die fehlende Nephrotoxizität des Kontrastmittels ist besonders vorteilhaft für die Evaluierung von Patienten mit Nierentransplantaten und Nierenspendern. Hier konnten sehr gute Ergebnisse im Vergleich zu den invasiveren Standardverfahren gewonnen werden $[9,25]$.

\section{Renale Läsionen}

Die räumliche Differenzierung feinster Strukturen wie z.B. die Detektion eines Zystenwandkarzinomes erscheint derzeit trotz Verwendung hochauflösender Sequenzen schwierig. Hier behält die Spiral-Computertomographie (SpiralCT) ihren Stellenwert. Die Magnetresonanztomographie kann jedoch aufgrund ihres besseren Gewebekontrastes, der selektiven Darstellung ver- 


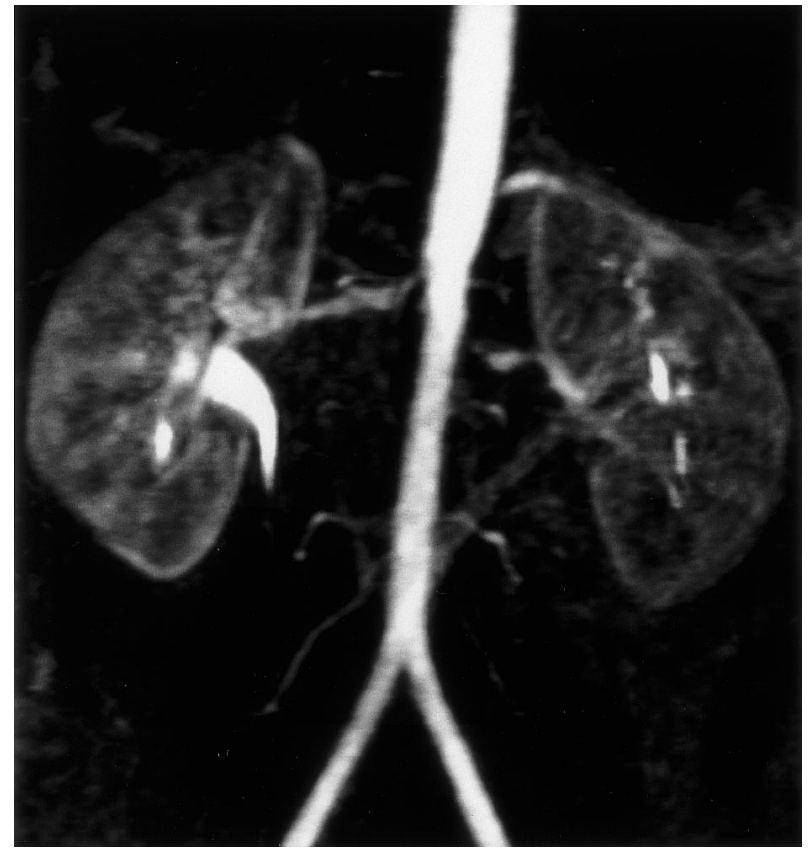

Abb. 5 Hochauflösende 3D-Gadolinium-MRA (TR=4,6 ms, TE=1,8 ms, Voxelgröße 0,7×1×1,1 mm) einer 11jährigen Patientin mit Hypertonus und Neurofibromatose. Diagnose: Hypoplastische Aorta mit beidseitiger ostialer Nierenarterienstenose und multiplen Kaliberschwankungen

Abb. $6 \nabla$ 63jähriger Patient mit Hufeisenniere. Die hochauflösende 3D-Gadolinium-MRA (TR=4,6 ms, $\mathrm{TE}=1,8 \mathrm{~ms}$, Voxelgröße $0,7 \times 1,2 \times 1,2 \mathrm{~mm}$ ) zeigt in der früharteriellen Phase neben den beiden Hauptnierenarterien noch zwei akzessorische Nierenarterien im Bereich der distalen Aorta oberhalb der Bifurkation sowie der A. iliaca communis rechts. Die bifurkationsnahe akzessorische Nierenarterie links (Bild rechts unten) weist eine mittelgradige ostiale Stenose auf. In der venösen Phase (Bild rechts oben) Darstellung der vollständig kontrastierten Hufeisenniere mit Nachweis multipler venöser Abflüsse. Diagnose: stenosierte akzessorische Nierenarterie bei Hufeisenniere schiedener Gewebeanteile einschließlich der Vaskularisation einen wichtigen Beitrag zur Differenzierung von Läsionen leisten [18]. Eine Erhöhung der Spezifität bei der Läsionsdifferenzierung läßt sich durch die Analyse der Kontrastierungskinetik in den einzelnen 3D Datensätzen der Multiphasenangiographie analog zur mehrphasigen SpiralCT erzielen. Dies soll anhand der häufigsten Pathologien exemplarisch dargestellt werden.

\section{Zysten}

Der Nachweis einer glatt begrenzten, dünnwandigen Struktur mit flüssigkeitsäquiintensem Binnensignal sowie fehlender Kontrastmittelanreicherung sichert die Diagnose einer Zyste [18] (Abb. 9). Die Diagnose kann aus der Analyse der T2 gewichteten HASTE Bilder, der Einzelschichten der multiphasischen 3D-Gd-MRA und der statischen fettunterdrückten FLASH-Aufnahmen nach Kontrastmittelgabe leicht gestellt werden. Anhand der Signalaussparung in den post KM-Aufnahmen lassen sich selbst kleinste Zysten von 3 bis $4 \mathrm{~mm}$ erkennen. Einfache Zysten beinhalten Flüssigkeit mit ähnlicher Signalgebung wie Urin, sind signalarm in der T1-Wichtung und lassen bei Aus-
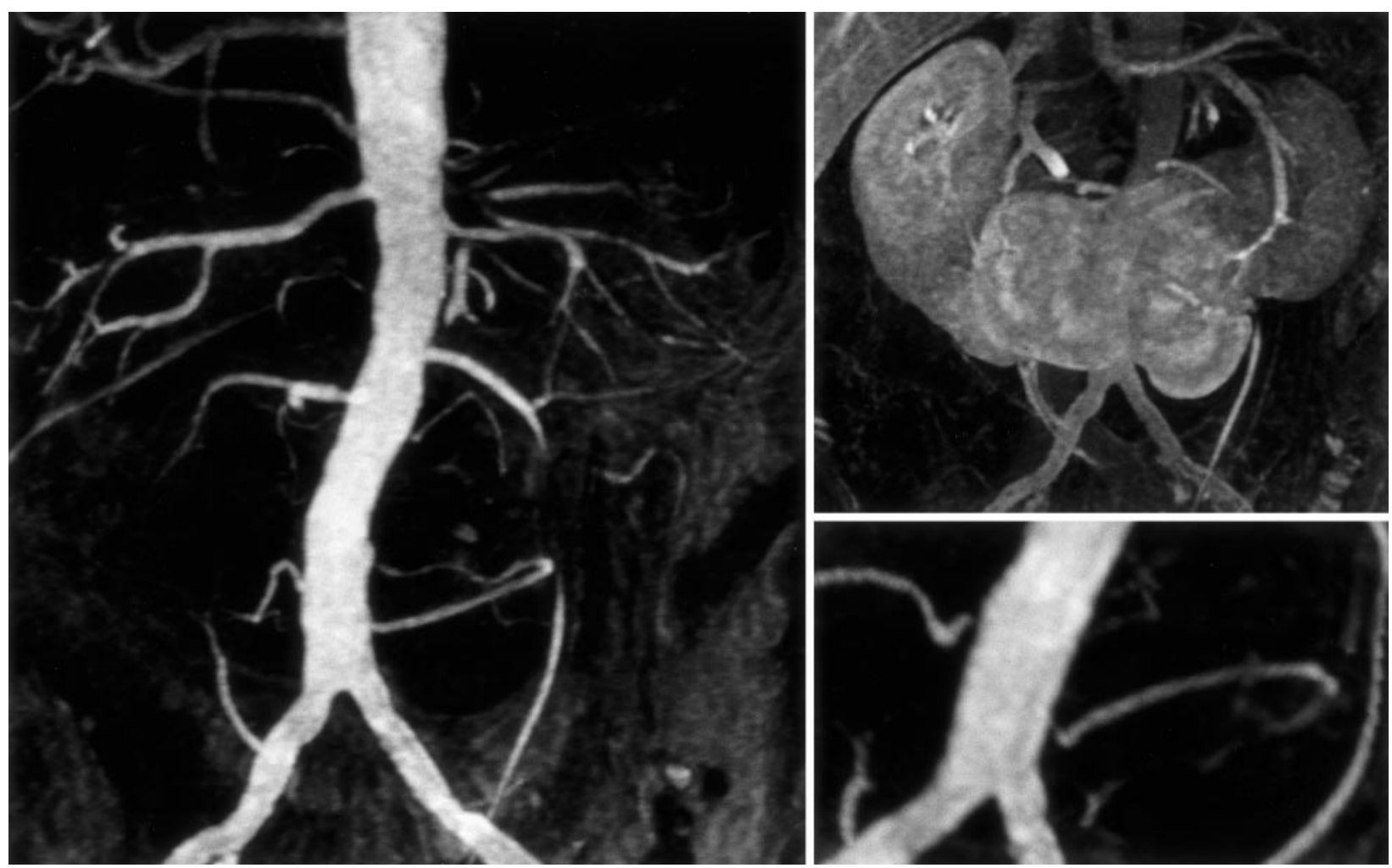


\section{Nierendiagnostik}
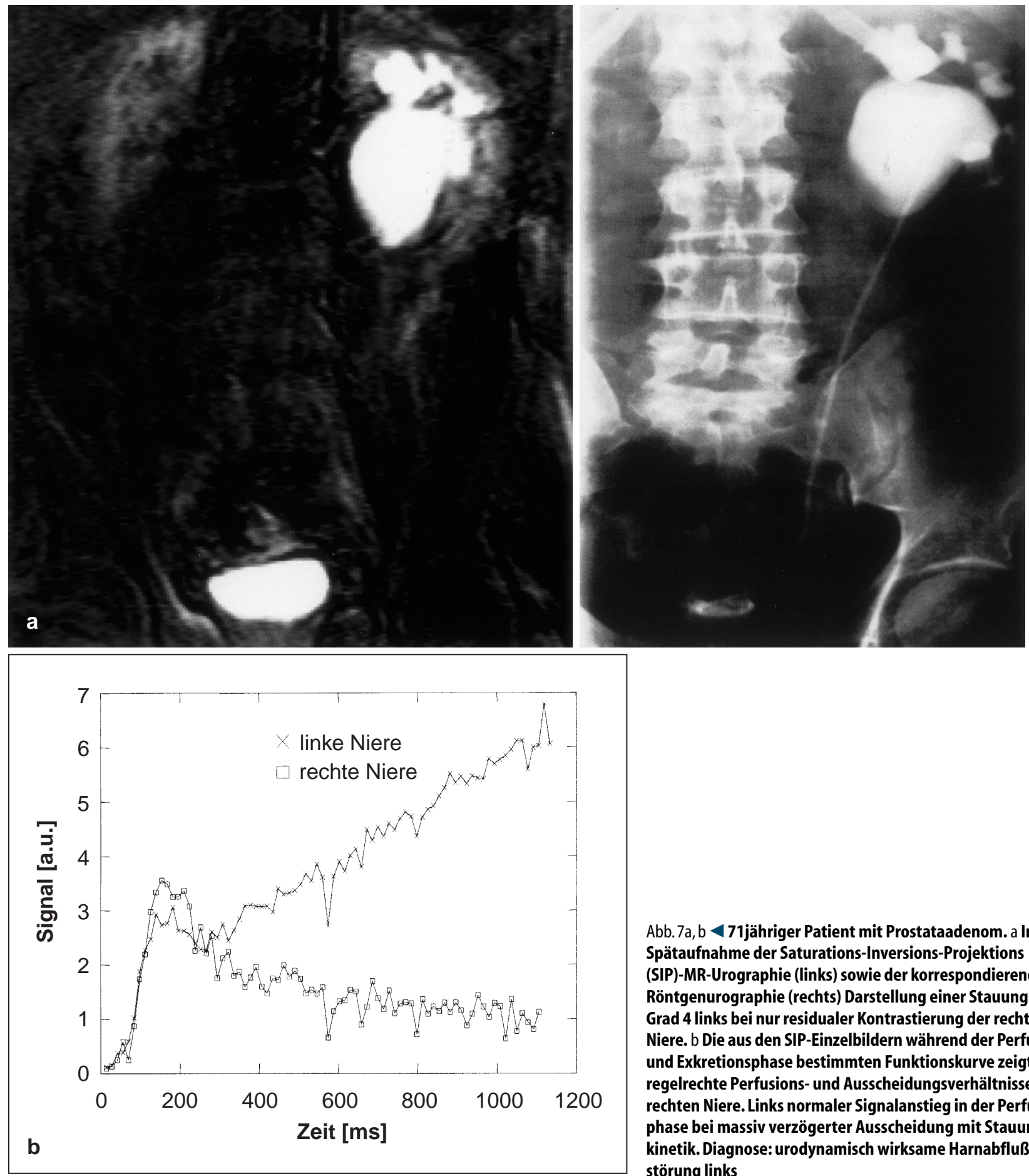

Abb. 7a, b < 71jähriger Patient mit Prostataadenom. a In der Spätaufnahme der Saturations-Inversions-Projektions (SIP)-MR-Urographie (links) sowie der korrespondierenden Röntgenurographie (rechts) Darstellung einer Stauungsniere Grad 4 links bei nur residualer Kontrastierung der rechten Niere. b Die aus den SIP-Einzelbildern während der Perfusionsund Exkretionsphase bestimmten Funktionskurve zeigt regelrechte Perfusions- und Ausscheidungsverhältnisse der rechten Niere. Links normaler Signalanstieg in der Perfusionsphase bei massiv verzögerter Ausscheidung mit Stauungskinetik. Diagnose: urodynamisch wirksame Harnabflußstörung links

dehnung außerhalb des Kortex keine eindeutige Wand erkennen. Komplizierte Zysten enthalten Blut, Septierungen oder Kalzifikationen. Die Magnetresonanztomographie ist im Vergleich zur CT sensitiver gegenüber diesen Änderungen des Gewebekontrastes. Selbst geringe Kalzifizierungen lassen sich als Signalverlust in den Gradienteneechobildern erkennen. Blut kann auch noch nach mehreren Wochen bis Monaten im subakuten Methämoglobinstadium als hyperintenses Areal in der T1-Wichtung bzw. chronischen Hämosiderinstadium in der T2-Wichtung erkannt werden. Organisierte Blutungen können Schwierigkeiten bei der Differenzierung gegenüber malignen Läsionen bereiten, deshalb sollte jede Kontrastmittelaufnahme in der Früh- und Spätphase nach mehreren Minuten ausgeschlossen werden.

Bei autosomal dominant vererbten polyzystischen Nieren findet sich eine progressive Zunahme multipler Nierenzysten in beiden Nieren, die im Spätstadium zur Niereninsuffizienz führen können (Abb. 10). Eine nichtinvasive Verlaufkontrolle der renalen Funktion ist hier wichtig. Häufig lassen 


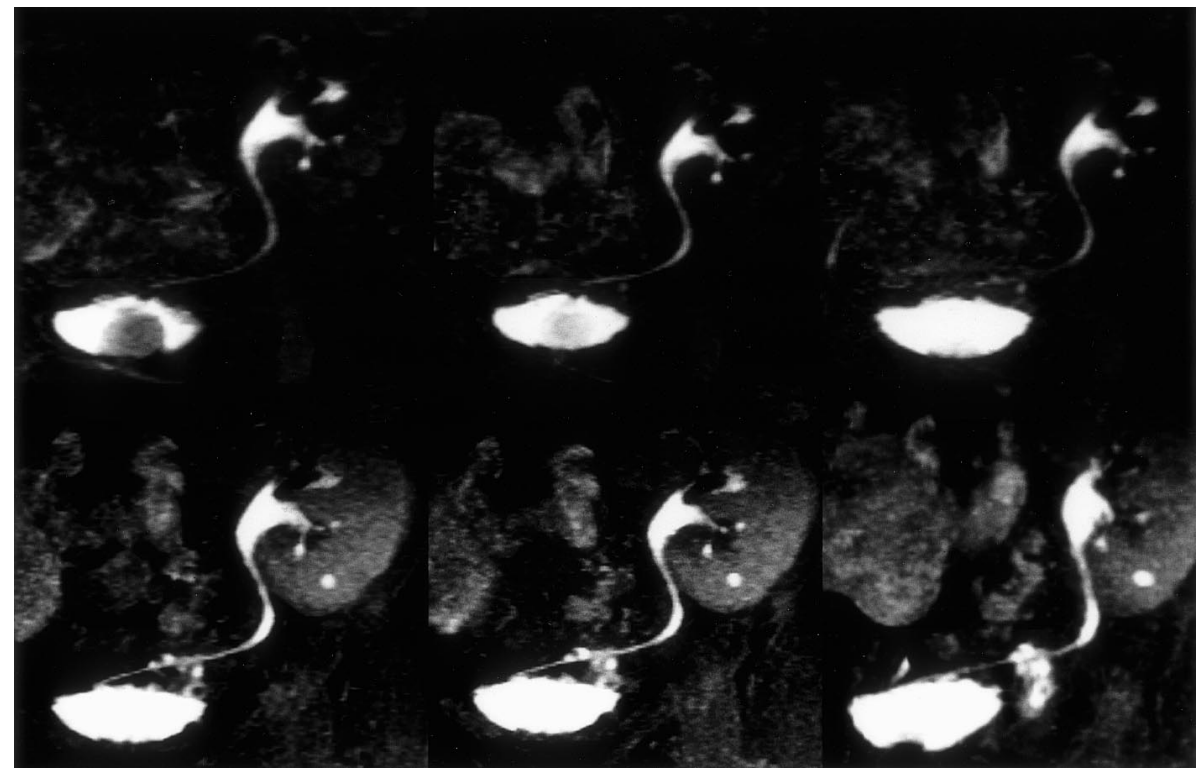

Abb. 8 43jähriger Patient nach Nierentransplantation. In der dynamischen MR-Urographie (mehrphasische 3D FLASH mit Fettunterdrückung, TR=5 ms, TE $=2 \mathrm{~ms}$, Meßzeit=27 s pro Phase, Zeit zwischen jeder Phase $=2$ min) Nachweis von Kontrastmittelausfluß (Gd-DTPA, Magnevist ${ }^{\circledR}$ ) im Bereich des distalen Ureterdrittels. Diagnose: Ureterverletzung bei Transplantatniere sich auch Zysten in der Leber, Niere und Pankreas nachweisen. Nach Diagnosestellung sollte auch eine MR Angiographie der cerebralen Arterien zum Ausschluß eines assoziierten sacciformen Aneurysmas durchgeführt werden.

\section{Angiomyolipome}

Angiomyolipome bestehen aus drei Elementen: Blutgefäße, glatte Muskulatur und Fett. Durch die starke Signalintensität in $\mathrm{T}_{1}$ gewichteten Sequenzen können bereits kleinste fettige Läsionen unter einem Zentimeter sensitiv nachgewiesen werden. Ein vollständiger Signalverlust in fettunterdrückten Aufnahmen beweist das Vorliegen von Fett. Überwiegen jedoch glatte Muskulatur- und Gefäßanteile kann die Ab- grenzung zum Nierenzellkarzinom schwierig werden.

\section{Nierenzellkarzinom}

Bei jedem soliden renalen Tumor ohne Nachweis fettiger Areale sollte an ein mögliches Nierenzellkarzinom gedacht werden. Die Genauigkeit der MRT bei der Detektion und Abgrenzung des Nierenzellkarzinoms wird in einigen Studien als vergleichbar zur CT angegeben [19-21]. Extrarenales Wachstum eines Nierenzellkarzinoms läßt sich in den hochauflösenden T2-gewichteten Sequenzen durch Suppression des periund pararenalen Fetts leicht erfassen (Abb. 11a). Bedingt durch grenzwertige Auflösung und Bewegungsartefakte gelingt eine Darstellung des Bezuges zur
Gerota-Faszie nicht in allen Fällen. Durch die multiplanare Schichtführung kann die Ausdehnung des Primärtumors in der kraniokaudalen Richtung besser erfaßt werden [21]. Deutlich heben sich auch retroperitonale Lymphknoten in diesen Sequenzen ab. Allerdings ist die Differenzierung zwischen hyperplastisch vergrößerten Lymphknoten und tumorös befallenen schwierig [22]. Nekrotische Lymphknoten werden von einigen Autoren als spezifisch für Lymphknotenbefall betrachtet, falls der Primärtumor ebenfalls nekrotisch ist.

Der große Vorteil besteht in der Beantwortung zusätzlicher Fragestellungen, insbesondere der vaskulären Versorgung (Abb. 11b, Abb. 12). Die Anwendung der 3D-Gd-MRA als Teil

Abb. 9 Charakterisierung von renalen Läsionen mittels schneller MR-Bildgebung. Darstellung einer in der T1-gewichteten FLASH

2D-Sequenz hypointensen, in der T2-gewichteten Turbospinechosequenz homogen hyperintensen Läsion am medialen Rand der linken Niere mit fehlender Begrenzung zum perirenalen Raum. Diagnose: einfache Nierenzyste
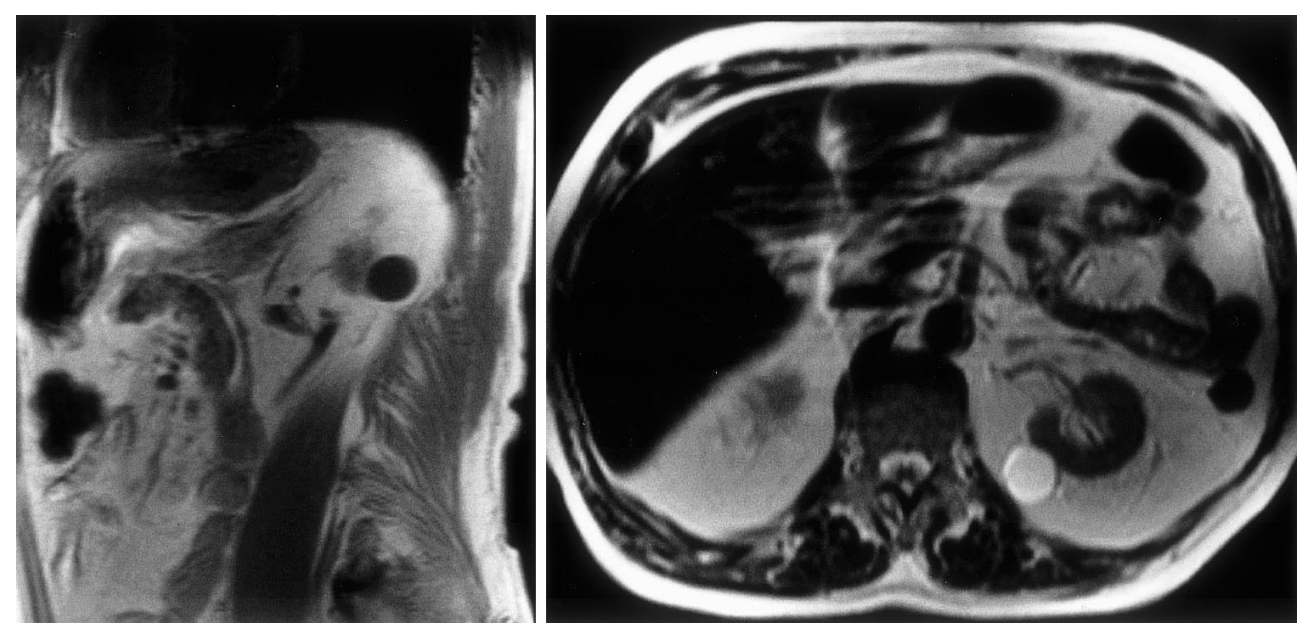


\section{Nierendiagnostik}
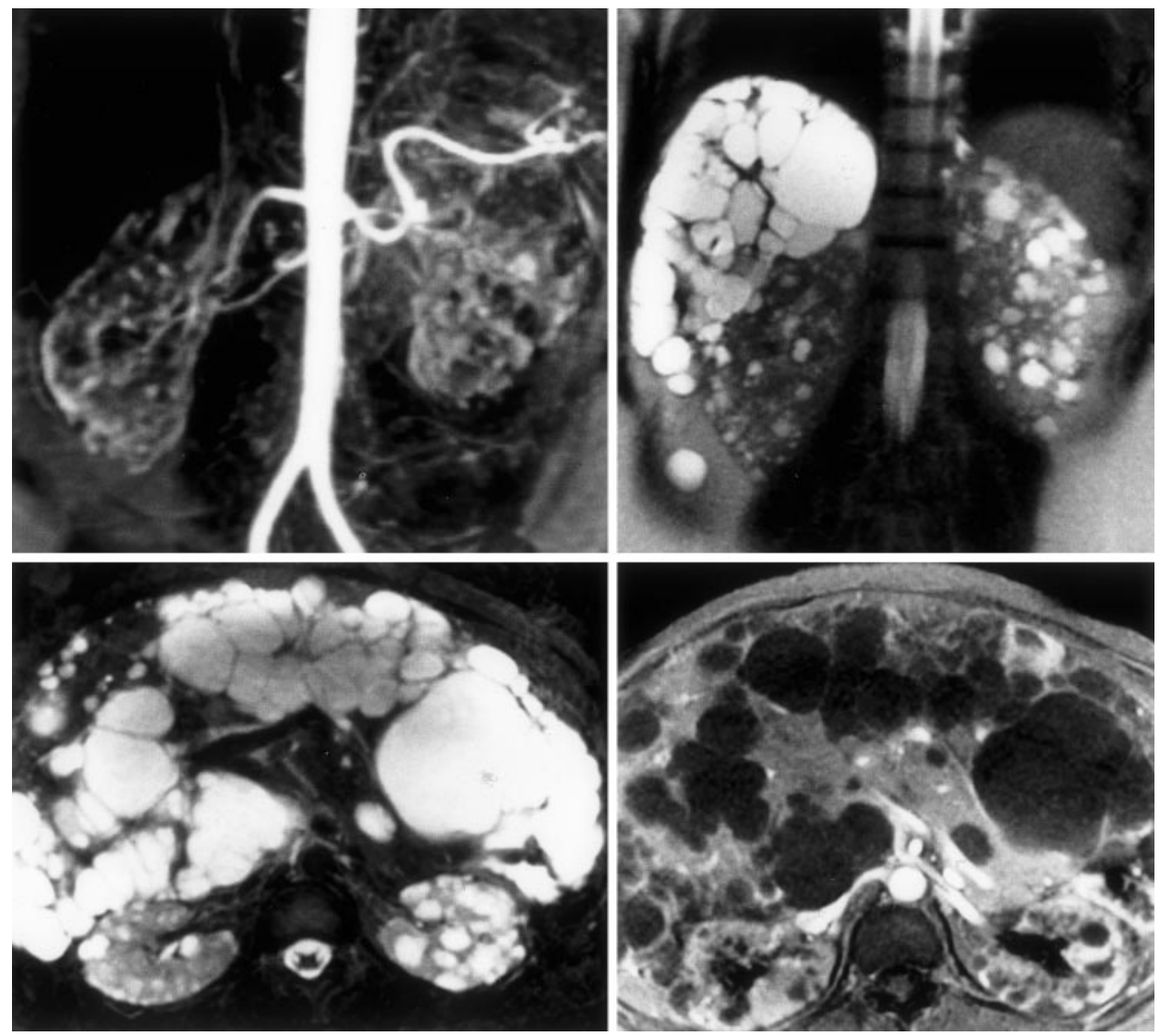

Abb. $10 \Delta$ 51jährige Patientin mit progressiver Niereninsuffizienz. Die 3D-Gadolinium-MRA (rechts oben) zeigt beidseits vergrößerte Nieren mit inhomogener Kontrastierung. In der hochauflösenden T2-gewichteten fettunterdrückten Multishot-Turbospinecho-Sequenz (rechts unten) und koronaren T2 gewichteten HASTE-Sequenz (links oben) Darstellung multipler Nierenzysten unterschiedlicher Größe mit teilweiser Signalabsenkung durch Einblutung. Nebenbefundlich multiple Leberzysten. Diagnose: autosomal dominante polyzystische Nierenerkrankung

eines umfassenden Untersuchungskonzepts erlaubt in hervorragender Weise die Beurteilung der Ausdehnung eines Nierenzellkarzinoms entlang der Nierenvene sowie der Vena cava inferior. Durch multiplanare Reformatierung des in der venösen Phase aufgenommenen $3 \mathrm{D}$-Datensatzes gelingt die exakte Beurteilung der Thrombusausdehnung sowie einer durch Tumoranteile bedingten venösen Gefäßkompression. Hierbei sollte auf die Kontrastmittelanreicherung eines Tumorthrombus in der Differenzierung zum Appositionsthrombus geachtet werden.

Neben diesen primären onkologischen Kriterien kommen eine Reihe funktioneller und technischer Aspekte hinzu. Hierzu gehören bei der Durchführung einer radikalen Tumornephrektomie die Beurteilung der kontralateralen Organfunktion unter Beurteilung der ipsi- und kontralateralen Nierenarterie (Abb.11b), der renalen Blutzufuhr, der parenchymalen Perfusion sowie der Ausscheidungsleistung und -kinetik (Abb. 11c). In einer Studie wurden $97 \%$ aller Nierenarterien der befallenen Niere mit der 3D-Gd-MRA erkannt [23]. Bei Durchführung einer partiellen Nephrektomie wird die Detektion verbleibenden funktionellen Restgewebes entscheidend. Wichtige technische Aspekte, die den Operationsvorgang beeinflussen, beinhalten die korrekte Identifikation akzessorischer Nierenarterien sowie den Nachweis kongenitaler Fehlbildungen des Urogenitaltraktes.

\section{Nierenlymphom}

Nierenlymphome sind üblicherweise Teil eines generalisierten Lymphombefalls, häufig im Rahmen eines NonHodgkin-Lymphoms. Der Lymphom- befall geht oft vom unmittelbar benachbarten Retroperitoneum aus. Charakteristisch ist der Erhalt der Nierenform bei massiv vergrößerten Nieren mit Verlust der kortikomedullären Differenzierung in den T1-gewichteten Bildern (Abb. 13). Nach Kontrastmittelgabe findet sich in der Frühphase im Gegensatz zum Nierenzellkarzinom eine insgesamt reduzierte Kontrastierung.

\section{Pathologien im Bereich der Harnabflußwege}

Sequentiell in der Perfusions- und Exkretionsphase aufgenommene $3 \mathrm{D}-\mathrm{Gd}-$ MRA oder SIP Datensätze ermöglichen eine dynamische Reno- und Urographie [24-26]. In den Bilderserien kann analog zur Röntgenurographie die Kontrastierung des Nierenparenchyms, der Kelchsysteme, des Nierenbeckens und der Ureteren im Seitenvergleich betrachtet werden. Verzögerte Kontrastierung des Kelchsystems, Tumoreinbruch in das Kelchsystem oder Nierenbecken (Abb. 11c), Abflußhindernisse mit Harnaufstau (Abb. 7) sowie Leckagen z.B. bei Verletzung der Ureteren 

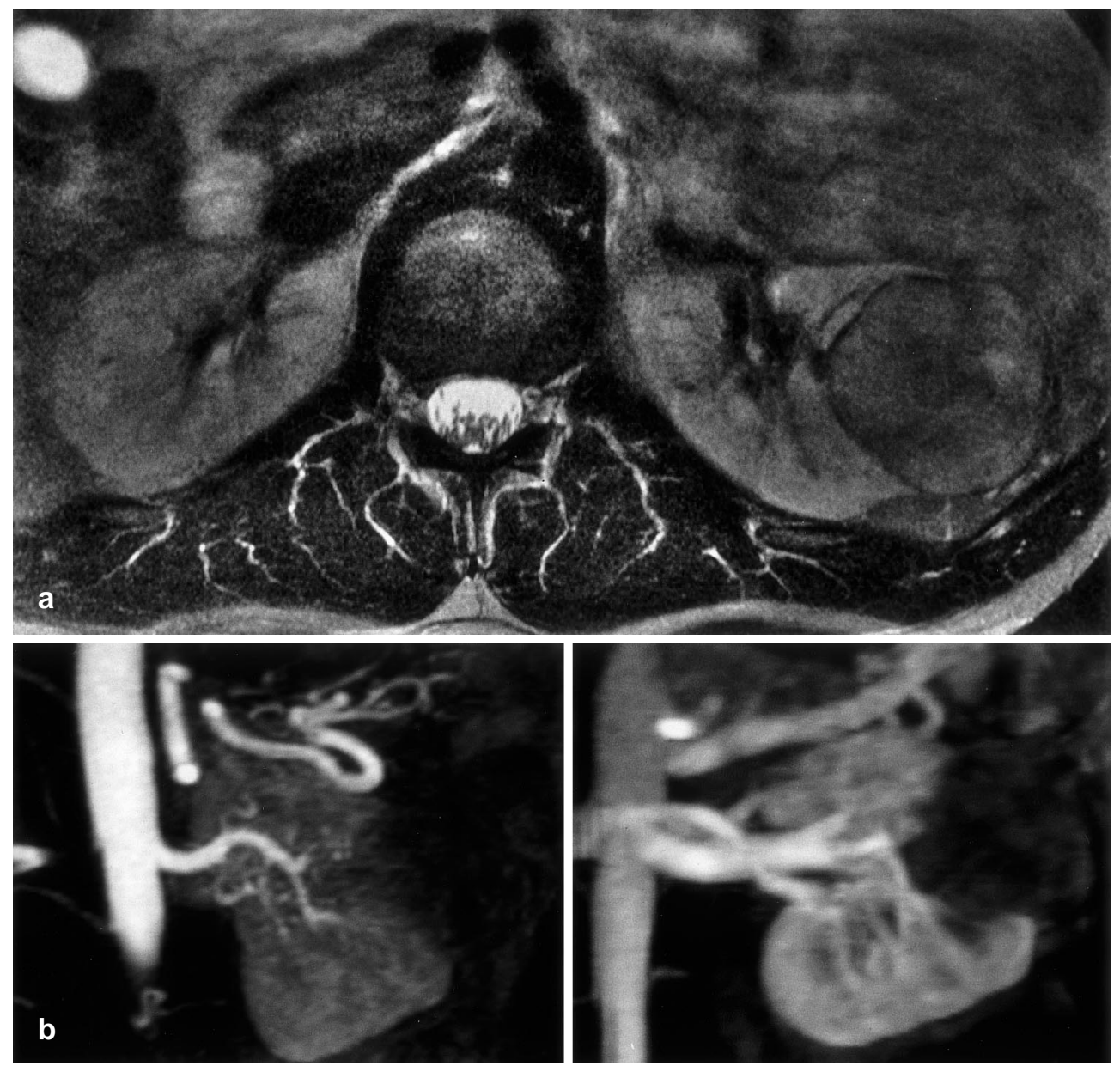

Abb.11a-c $<28$ jähriger Patient mit sonographisch diagnostiziertem linksseitigem Nierentumor. a In der hochauflösenden axialen

T2-gewichteten Turbospinechosequenz kein Einbruch des Tumors in den perirenalen Raum bei erhaltener Nierenkapsel. Kein Nachweis vergrößerter retroperitonealer Lymphknoten. $b$ In der arteriellen Phase der multiphasischen 3D-Gd-MRA (links) stellt sich der Tumor deutlich hypovaskularisiert dar mit Nachweis feiner fadenförmiger Kontrastierungen. In der venösen Phase (rechts) kein Anhalt für Tumorausdehnung entlang der Nierenvene. C Die MR-Urographie mittels einer SIP-Sequenz zeigt die Kompression des oberen Anteils des Kelchsystems in der linken Niere, kontralateral finden sich normale Abflußverhältnisse. Der Patient wurde einseitig nephrektomiert. Diagnose: hypovaskularisiertes Nierenkarzinom (T2, NO, MO, G2)

(Abb. 8) können somit erkannt werden. Die kontrastmittelverstärkte MR-Urographie sollte grundsätzlich mit der nativen Urographie mittels stark T2-gewichteten Sequenzen, z.B. HASTE, kombiniert werden. Bei Harnaufstau mit reduzierter Kontrastmittelaus- scheidung läßt sich so die Hydronephrose und Ureterdilatation nachweisen. Aufgrund der Signalauslöschung durch Suzeptibilitätsartefakte ist ein Steinnachweis allerdings nur indirekt durch Kontrastmittelaussparung oder Harnaufstau möglich.
Ein wesentlicher Vorteil der dynamischen MR-Urographie gegenüber der Röntgenurographie besteht darin, die Ausscheidungskinetik der Niere durch Analyse der Signalintensitäten in den einzelnen Datensätzen semiquantitativ darzustellen [26]. In den resultie- 


\section{Nierendiagnostik}
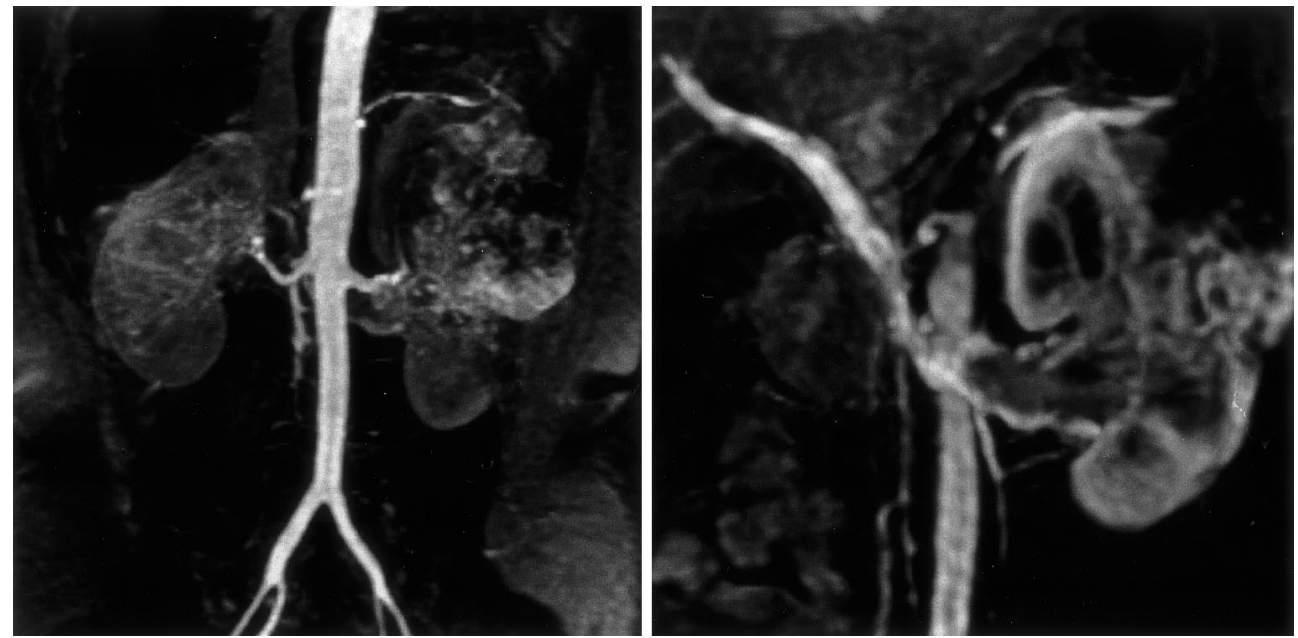

Abb. $12 \triangle 58$ jähriger Patient mit Hämaturie. In der arteriellen Phase der 3D-Gadolinium-MRA Darstellung einer inhomogen hypervaskularisierten Raumforderung im Bereich des Oberpols der linken Niere. In der venösen Phase Nachweis eines Tumorthrombus in der Nierenvene mit kräftiger wandständiger Kontrastierung. Diagnose: Nierenzellkarzinom mit Tumorthrombus (mit freundlicher Genehmigung von Seminars in Interventional Radiology)

renden Zeit-Intensitätskurven lassen sich analog zur Szintigraphie bereits geringe Änderungen im Kurvenverlauf nachweisen (Abb. $7 \mathrm{~b})$. Abflußhindernisse können somit auf ihre urodynamische Relevanz hin analysiert werden.

\section{Kongenitale Fehlbildungen}

Kongenitale Fehlbildung können sowohl die Gefäßversorgung der Niere, die Niere selbst als auch die ableitenden Harnwege betreffen. In einem umfassenden MR-Untersuchungskonzept kann jede dieser Pathologien erfaßt werden. Häufig finden sich multiple Nierenarterien (Abb. 6). Hier ist die koronare Orientierung des 3D-Gd-MRA Datensatzes von Vorteil, da die überzähligen Nierenarterien entlang der gesamten abdominellen Aorta sowie der Beckenarterien entspringen können [27]. Bei Kindern findet sich in Assoziation mit der Neurofibromatose gelegentlich eine entwicklungsbedingte Hypoplasie der proximalen Nierenarterien, oft in Kombination mit einer Aortenkoarktation oder Hypoplasie (Abb. 5). Häufig lassen sich multiple stenosierte Nierenarterien nachweisen. Fehlanlagen der Niere sowie des Harntraktes sind durch Kombination aus morphologischer Bildgebung und MR-Urographie leicht diagnostizierbar. Eine verbleibende diagnostische Herausforderungen stellen diskrete Abnormalitäten der Ureteren wie z.B. Ureterstenosen, Doppelungen oder
Hypoplasien dar, da hier u.U. die Auflösung der $3 \mathrm{D}-\mathrm{Gd}-\mathrm{MRA}$-Sequenzen nicht ausreichend ist.

\section{Diskussion}

Die Magnetresonanztomographie war traditionell im Abdomen durch multiple Artefakte limitiert und wurde daher nur sehr beschränkt eingesetzt. Schnellere MR-Systeme mit leistungsfähige- ren Gradientensystemen erlauben eine drastische Reduktion der Meßzeit und damit eine deutliche Verbesserung der abdominellen Bildgebung. Dennoch bleibt die Auflösung und Geschwindigkeit konventioneller radiologischer Verfahren unerreicht. Ein wesentlicher Vorteil der Magnetresonanztomographie ist jedoch die Kombination mehrerer ultraschneller Untersuchungsverfahren zur Analyse von Morphologie, Hämodynamik und Funktion. Im Bereich der Niere lassen sich durch Integration dieser unterschiedlichen Information Pathologien der Gefäßversorgung, des Nierenparenchyms sowie der Harnabflußwege mit hoher diagnostischer Ge-

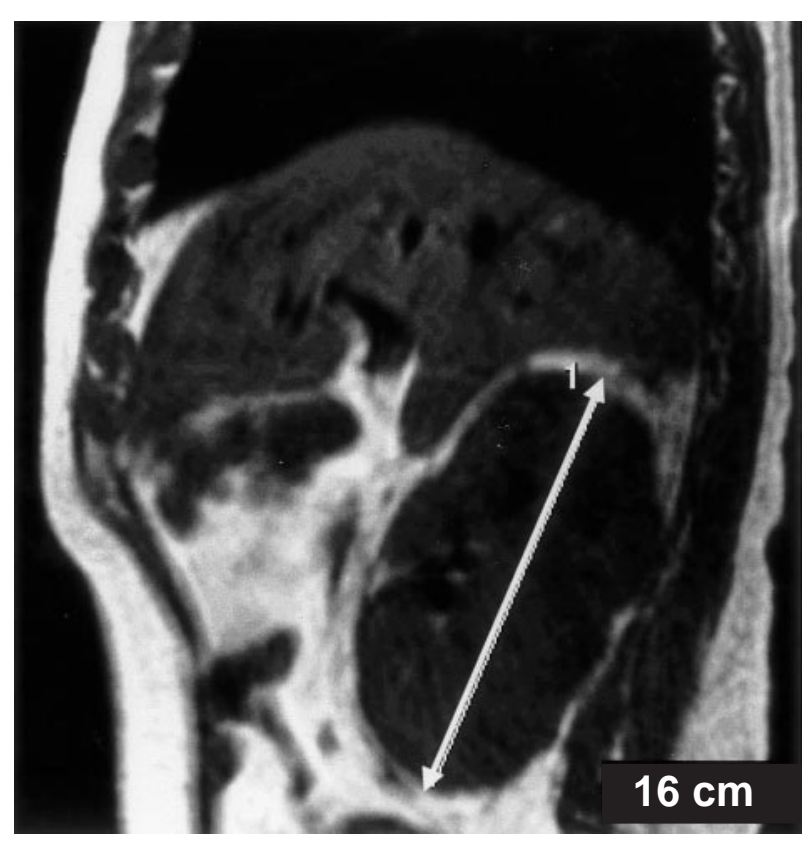

Abb. $13 \triangle$ 42jähriger Patient mit palpatorisch vergrößerter Niere. Im T1-gewichteten Spinecho-Bild Nachweis einer massiv vergrößerten Niere bei erhaltener Nierenform. Aufhebung der kortikomedullären Differenzierung sowie Unterbrechung der Fettkapsel. Diagnose: Nierenlymphom 
nauigkeit abklären. Dieser kombinierte Ansatz ist trotz der höheren Untersuchungskosten der Magnetresonanztomographie gegenüber der Gesamtheit konventioneller Untersuchungsverfahren als kosteneffektiv zu betrachten. Zusätzlich entsteht für den Patienten keine Exposition gegenüber ionisierenden Strahlen oder nephrotoxischen Kontrastmitteln. Die Rolle der konventionellen Verfahren wird sich demgegenüber zukünftig auf akute Fragestellungen in der Traumatologie, der therapeutischen Intervention sowie präoperativer Abklärung mit hohen Anforderungen an räumlicher Auflösung beschränken.

Die Autoren danken Prof. Dr. J.H. Clorius für die hilfreichen Diskussionen, Ch. Konold und J. Wiegand für die gewohnt gute Qualität der photographischen Abbildungen.

\section{Literatur}

1. McKinnon GC (1998) The physics of ultrafast magnetic resonance imaging. In: Debatin JF, McKinnon GC (eds) Ultrafast MRI.Springer, Berlin Heidelberg New York, pp 1-51

2. Stehling MK, Nitz W, Holzknecht N (1995) Fast and ultra-fast magnetic resonance tomography. Basic principles, pulse sequences and special properties. Radiologe 35:879-893

3. Cavagna FM, Maggioni F, Castelli PM, Dapra M, Imperatori LG, Lorusso V, Jenkins BG (1997) Gadolinium chelates with weak binding to serum proteins. A new class of highefficiency, general purpose contrast agents for magnetic resonance imaging. Invest Radiol 32:780-896

4. Knopp MV, Obier C, Zuna I, Junkermann $\mathrm{H}$, Heß T, Junkermann I, Brix G, van Kaick G (1998) Multi-reader-Analyse zur Beurteilung der funktionellen MR-Mammographie. Radiologe 38:307-314

5. Prince MR (1994) Gadolinium-enhanced MR aortography. Radiology 191:155-164

6. Schoenberg SO, Knopp MV, Prince MR, Londy FJ, Knopp MA (1998) Arterial phase 3D Gadolinium MRA of the renal arteries: strategies for timing and contrast media injection. Invest Radiol 33:506-514
7. Li W, Chavez D, Prasad PV, Edelman RR (1997) Magnetic resonance urography by breathhold contrast-enhanced three-dimensional FISP. J Magn Reson Imaging 7:309-311

8. Oesingmann N, Schad LR; Knopp MV, Dörsam J, Lorenz WJ (1995) Magnetic resonance urography using the SIP-SE sequence. ISMRM 1487

9. Stafford Johnson DB, Lerner CA, Prince MR, Kazanjian SN, Narasimham DL, Leichtman AB, Cho KJ (1997) Gadolinium-enhanced magnetic resonance angiography of renal transplants. Magn Reson Imaging 15:13-20

10. Schoenberg SO, Just A, Bock M, Knopp MV, Persson PB, Kirchheim HR (1997) Non-invasive analysis of renal artery blood flow dynamics with MR CINE phase-contrast flow measurements. Am J Physiol 272:H2477-H2484

11. Schoenberg SO, Knopp MV, Bock M, Kallinowski F, Just A, Essig M, Hawighorst $H$, Schad LR, van Kaick G (1997) Renal artery stenosis: grading of hemodynamic changes with MR CINE phase-contrast flow measurements. Radiology 203:45-53

12. Bock M, Schoenberg SO, Schad LR, Knopp MV, Essig M, van Kaick G (1998) Intervealed gradient echo planar (IGEPI) and phase contrast (CINE-PC) flow measurements in the renal artery. JMRI 8:889-895

13. Schoenberg SO, Knopp MV, Bock M, Prince MR, Allenberg JR (1998) Combined morphologic and functional assessment of renal artery stenosis using gadolinium enhanced magnetic resonance imaging. Nephrol Dial Transplant 13:2738-2742

14. Schoenberg SO, Bock M, Knopp MV, Essig M, Laub G, Hawighorst H, Zuna I, Kallinowski F, van Kaick G (1999) Renal arteries: optimization of 3D gadolinium MR angiography with bolus-timing independent ultrafast multiphase acquisition in a single breath hold. Radiology (in press)

15. Prince MR, Grist TM, Debatin JF (1998) Renal arteries. In:Prince MR, Grist TM, Debatin JF (eds) 3D contrast MR angiography, 2nd ed.Springer, Berlin Heidelberg New York, pp 89-105

16. Prince MR, Schoenberg SO, Ward J, Londy F, Wakefield T, Stanley J (1997)

Hemodynamically significant atherosclerotic renal artery stenosis: MR angiographic features. Radiology 205:128-136

17. Wasser MN, Westenberg J, van der Hulst VPM, van Baalen J, van Bockel JH, van Erkel AR, Pattynama PMT (1997) Hemodynamic significance of renal artery stenosis: digital subtraction angiography versus systolically gated three-dimensional phase-contrast MR angiography. Radiology 203:333-338
18. Semelka RC, Kettritz U, Brown ED (1996) Kidneys, adrenal glands and retroperitoneum. In:Edelman RR, Hesselink JR, Zlatkin MB (eds) Clinical magnetic resonance imaging, 2nd ed. Saunders, Philadelphia, pp 1513-1535

19. Semelka RC, Shoenut JP, Kroeker MA, Kroeker MA, MacMahon RG, Greenberg HM (1992) Renal lesions: controlled comparison between $\mathrm{CT}$ and 1.5-T MR imaging with nonenhanced and gadoliniumenhanced fat-suppressed spin-echo and breath-hold FLASH techniques. Radiology 182:425-430

20. Semelka RC, Shoenut JP, Magro CM, Kroeker MA, MacMahon R, Greenberg HM (1993) Renal cancer staging: comparison of contrastenhanced CT and gadolinium-enhanced fat-suppressed spin-echo and gradientecho MR imaging. MRI 3:597-602

21. Hallscheidt P, Stolte E, Roeren T, Pomer S, Drehmer I, Kauffmann GW (1998) The staging of renal-cell carcinomas in MRT and CT a prospective histologically controlled study. Rofo 168:165-170

22. Studer UE, Scherz S, Scheidegger J, Kraft R, Sonntag R, Ackermann D, Zingg EJ (1990) Enlargement of regional lymph nodes in renal cell carcinoma is often not due to metastases. J Urol 144:243-245

23. Choyke PL, Walther MM, Wagner JR, Rayford W, Lyne JC, Linehand WM (1997) Renal cancer: preoperative evaluation with dual-phase three-dimensional MR angiography. Radiology 205_767-771

24. Prasad PV, Chen QC, Goldfarb JW, Priatna A, Edelman RR (1998) Functional assessment of renal artery stenosis by captopril MR renography. Radiology 209(P):490

25. Low RN, Martinez AG, Steinberg SM, Alzate GD, Kortman KE, Bower BB, Dwyer WJ, Prince SK (1998) Potential renal transplant donors: evaluation with gadolinium-enhanced MR angiography and MR urography. Radiology 207:165-172

26. Knopp MV, Dörsam J, Oesingmann N, Piesche $S$, Hawinghorst $H$, Wiesel M, Schad LR, van Kaick G (1997) Funktionelle MR-Urographie bei Patienten mit Nierentransplantaten. Radiologe 37:233-238

27. Schoenberg SO, Prince MR, Knopp MV, Allenberg JR (1998) Renal MR angiography. In: Body MR angiography. Magn Reson Clin North Am 6:351-370 